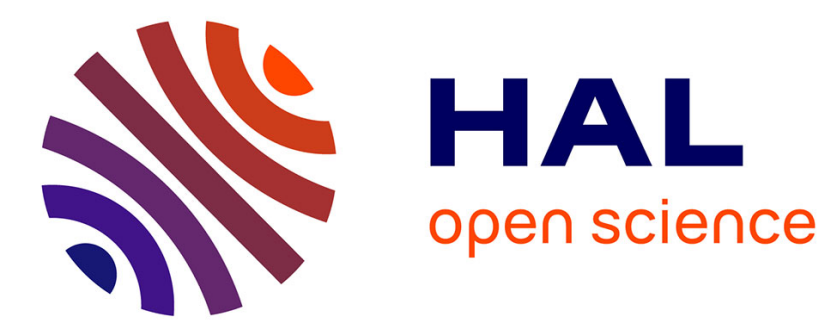

\title{
Interfacial properties of charge asymmetric ionic liquids
}

\author{
Jose Alejandre, Fernando Bresme, Minerva Gonzalez-Melchor
}

\section{To cite this version:}

Jose Alejandre, Fernando Bresme, Minerva Gonzalez-Melchor. Interfacial properties of charge asymmetric ionic liquids. Molecular Physics, 2009, 107 (04-06), pp.357-363. 10.1080/00268970902780270 . hal-00513257

\section{HAL Id: hal-00513257 \\ https://hal.science/hal-00513257}

Submitted on 1 Sep 2010

HAL is a multi-disciplinary open access archive for the deposit and dissemination of scientific research documents, whether they are published or not. The documents may come from teaching and research institutions in France or abroad, or from public or private research centers.
L'archive ouverte pluridisciplinaire HAL, est destinée au dépôt et à la diffusion de documents scientifiques de niveau recherche, publiés ou non, émanant des établissements d'enseignement et de recherche français ou étrangers, des laboratoires publics ou privés. 


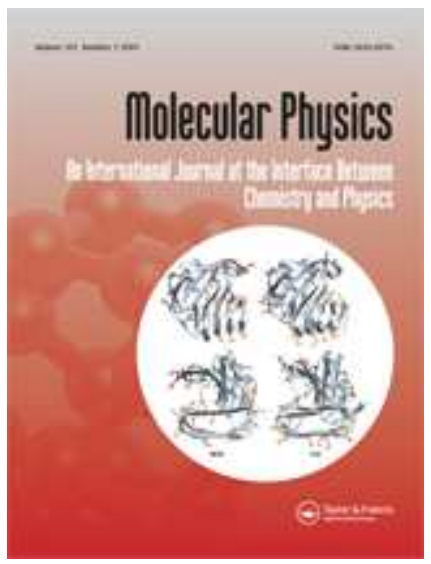

\section{Interfacial properties of charge asymmetric ionic liquids}

\begin{tabular}{|r|l|}
\hline Journal: & Molecular Physics \\
\hline Manuscript ID: & TMPH-2008-0402.R1 \\
\hline Manuscript Type: & Special Issue Paper - Dr. Jean-Jacques Weis \\
\hline Author: & 12-Jan-2009 \\
\hline Complete List of Authors: & $\begin{array}{l}\text { Alejandre, jose; Universidad Autonoma } \\
\text { Metropolitana-Iztapalapa, Departamento di Quimica; Universidad } \\
\text { Autonoma, Departamento di Quimica } \\
\text { Bresme, Fernando; Imperial College London, Chemistry; Fernando } \\
\text { Bresme, Fernando Bresme } \\
\text { Gonzalez-Melchor, Minerva; Universidad Autonoma de Puebla, } \\
\text { Instituto de de Fisica }\end{array}$ \\
\hline Keywords: & $\begin{array}{l}\text { Computer Simulations, Ionic liquids, Coexistence properties, } \\
\text { Interphase potential, Restricted Primitive model }\end{array}$ \\
\hline $\begin{array}{l}\text { Note: The following files were submitted by the author for peer review, but cannot be converted } \\
\text { to PDF. You must view these files (e.g. movies) online. }\end{array}$ \\
\hline \begin{tabular}{l} 
asymmetric-revised.tex \\
\hline
\end{tabular}
\end{tabular}

\section{scholarONE" \\ Manuscript Central}




\title{
Interfacial properties of charge asymmetric ionic liquids
}

\author{
José Alejandre $^{1}$, Fernando Bresme ${ }^{2}$ and Minerva González-Melchor ${ }^{3}$
}

(January 12, 2009)

(1) Departamento de Química, Universidad Autónoma Metropolitana-Iztapalapa, Av. San Rafael Atlixco 186, Col. Vicentina, 09340 México D.F., México

(2) Department of Chemistry, Imperial College London, Exhibition Road, London, SW7 2AZ, UK

(3) Instituto de de Física, Universidad Autónoma de Puebla, Apartado Postal J-48, 72570, Puebla, México

\begin{abstract}
We report molecular dynamics simulations of the coexistence and interfacial properties of ionic liquids as a function of cation/anion, $\left(z_{+}: z_{-}\right)=(2:-1)$, (4:1 ), charge asymmetry. Our results correct previous computations of the coexistence curve of (2:-1) charge asymmetric systems, obtained via the fine-lattice discretization method. In agreement with previous computations we report a reduction in the critical temperature and an increase in the critical density with charge asymmetry. We have quantified the interphase potential resulting from the charge asymmetry, by analyzing the charge density across the liquid-vapor interface. We show that the Debye-Hückel theory predicts reasonably well the magnitude and temperature dependence of the interphase potential for moderate charge asymmetries (2:-1) but it underestimates, by about $50 \%$, the electrostatic potential of ionic liquids with high charge asymmetries (4:-1).
\end{abstract}

Paper submitted for the J.J. Weis Special Issue 


\section{Introduction}

The calculation of the coexistence and interfacial properties of ionic fluids, including asymmetry in size and in charge has been a subject of great interest in the last decade. ${ }^{1-9}$ Asymmetry is an essential characteristic of many charged fluids, colloids, proteins and ionic liquids. It has been shown that charge and size asymmetry strongly influence the coexistence phase diagram of simple ionic liquids. This notion can be exploited to control mixing in multicomponent mixtures for instance. In order to describe the phase behavior of these systems it is therefore necessary to gain fundamental insight on how the magnitude and spatial distribution of charges control the mutual interactions between simple ionic liquids and complex macromolecular structures.

Electrostatic interactions provide many opportunities to organize matter in an effective way. The fact that positive and negative charges are present, and also that their effect can be tuned either by changing particle diameter or charge magnitude, opens the route to create complex structures using simple building blocks. A recent illustration of this notion is the self assembly of ionic colloidal crystals. ${ }^{10}$ Some crystalline phases observed in these experiments were first predicted in computer simulations of the restricted primitive model (RPM), ${ }^{11}$ in which, equal-size hard spheres interact through Coulombic interactions. Those results were later confirmed and refined in additional simulations of the restricted primitive model. ${ }^{12,13}$

The RPM as well as other versions of this model that incorporate size and charge asymmetries, have been extensively investigated using computer simulations and theory. These models enable systematic analyses of the dependence of the phase coexistence properties of ionic fluids with Coulombic interactions. The restricted primitive model in particular, has provided answers to long standing questions regarding the nature of the criticality of ionic system. ${ }^{14-16}$ This simple model has also provided considerable insight on the structure of ionic liquids. It is now well accepted that one essential characteristic of ionic fluids is ion association into clusters of different sizes. Ion clustering has been extensively investigated using computer simulations., ${ }^{5,17-20}$ The first study of clustering near the critical point was reported by Prof. Weis and co-workers. ${ }^{19}$ This work showed vivid evidence that near the critical point ions organize into clusters that are globally neutral. Clustering, can strongly influence the coexistence properties, since the interactions between ions and clusters are weakened as compared with the bare ion-ion Coulombic 
interaction. Indeed, the strong changes in critical temperature and density observed as a function of charge and size asymmetry can be rationalized in terms of ion clustering. Ion association is also an essential element of the Fisher and Levin theory, which provides a quantitative representation of the coexistence properties of the RPM near the critical point. ${ }^{21}$

In this paper we focus on the interfacial properties of charge asymmetric ionic liquids. The phase diagram and critical behavior of charge- and size-asymmetric fluids has been investigated using Monte Carlo and Molecular Dynamics simulations. ${ }^{1,3-7,20,22-24}$ Aqua et. al. ${ }^{25}$ investigated the criticality in charge asymmetric fluids, $\xi=z_{+} / z_{-}=2$ and 3. They considered a theory based on the generalization of the Debye-Hückel approach, which includes ion association (from dimers to tetramers), cluster solvation by charged ions and hard core interactions. This theory showed qualitative agreement with the coexistence curve obtained using the fine-lattice discretization simulation method. All the investigations referred to above agree in a number of observations: a) Asymmetry in size and charge strongly affect the critical parameters. The reduced critical temperature decreases and the critical density increases with increasing charge asymmetry.This observation is in contradiction with the predictions of the Mean Spherical approximation. b) The vapor phase is structured in a small fraction of free ions and mostly neutral clusters, trimers, tetramers or pentamers, for $\xi=2,3$ and 4 asymmetries respectively. ${ }^{5,20,26}$ This situation is reminiscent of the clustering studies of the RPM, where the dominant species are dimers. ${ }^{19}$

In comparison to the study of critical properties, the investigation of the interfacial properties and the coexistence diagram of charge asymmetric ionic fluids has deserved less attention. Asymmetry introduces new and interesting phenomenology. As a matter of fact results in a violation of local electroneutrality at the interface, which results in an interphase electrostatic potential, or Galvani potential. This potential has been investigated in size asymmetry mixtures using computer simulations, ${ }^{4}$ and in charge-asymmetric ionic fluids using the Debye-Hückel theory and a modified Bjerrum approach. ${ }^{25}$ These theories predict a significant electrostatic potential that increases with charge asymmetry. To the best of our knowledge the accuracy of these theories has not been tested against simulation data.

In this work we extent our investigations on the interfacial properties of ionic liquids to charge asymmetric fluids. We report new results on the coexistence properties of 
these systems, namely: phase coexistence curve, interfacial structure, and interphase electrostatic potential. We further discuss the accuracy of recent theoretical approaches in describing the electrostatic properties of these charged interfaces.

\section{Potential model and simulations details}

We have investigated ionic liquids consisting of ions of equal diameter, $\sigma$, and different charge. The system contains $N=N_{+}+N_{-}$ions, $N_{+}$carrying positive charge $q_{+}=z_{+} e$ and $N_{-}$a negative charge $q_{-}=-e$, where $e$ is the electron charge. The whole system is electrically neutral. The asymmetry is defined in terms of the parameter, $\xi=N_{+} / N_{-}=$ $z_{+} / z_{-}$. Two ions interact according to the following potential,

$$
u(r)=U_{R}(r)+\frac{1}{4 \pi \varepsilon_{0}} \frac{q_{+} q_{-}}{r}
$$

where $U_{R}(r)$ is the repulsive contribution, $r$ the distance between two ions and $\epsilon_{0}$ is the vacuum permittivity. We have considered two models for the repulsive part, a hard core model,

$$
U_{R}(r)= \begin{cases}+\infty, & r<\sigma \\ 0 & r \geq \sigma\end{cases}
$$

and the Soft Primitive Model (SPM), where the hard core repulsion is replaced by a soft interaction, ${ }^{1,3}$

$$
U_{R}(r)=A\left(\frac{\sigma}{r}\right)^{n}
$$

where the exponent, $n$, was set to $n=225$, and the constant $A$,

$$
A=\frac{\left|z_{i} z_{j}\right| e^{2}}{4 \pi \varepsilon_{0} \sigma}
$$

follows from the condition $u(\sigma)=0 .^{3}$

Throughout the paper we use reduced units. The length is reduced by the ion diameter, $\sigma$, and the energy by the minimum, $\epsilon$, of the potential between unlike ions. For the hard core model this is given by, $\epsilon=\left|z_{+} z_{-}\right| e^{2} /\left(4 \pi \epsilon_{0} \sigma\right)$, and for the soft repulsive model $\epsilon=u_{+-}\left(r_{\min }\right)=\left|A\left[n^{n /(1-n)}-n^{1 /(1-n)}\right]\right|$, where the bars indicate absolute value, and $r_{\text {min }}=n^{1 /(n-1)} \sigma$, defines the location of the minimum of the potential energy curve. ${ }^{3}$ The 
reduced temperature is then defined as, $T^{*}=k_{B} T / \epsilon$, where $k_{B}$ is the Boltzmann constant and $T$ the temperature. We showed in our previous work that the SPM predicts virtually the same coexistence properties as the hard core RPM. ${ }^{1,3}$ We will show here that similar conclusions apply to charge asymmetric ionic fluids.

The simulations of the hard core model were performed using canonical Monte Carlo simulations, similar to those reported in refs. ${ }^{1,11,19}$ These computations consisted of 1296 cations and 648 anions for $\xi=2$ asymmetry. The maximum displacement of the ions was adjusted to give acceptance rates of the order of $50 \%$. The simulations involved several blocks each consisting of $2-4 \times 10^{5}$ steps of equilibration and up to $5 \times 10^{5}$ steps of production. The soft core model was simulated using constant temperature Molecular Dynamics ${ }^{27-29}$ with a multiple time step algorithm. ${ }^{30}$ The system sizes, $\left(\mathrm{N}_{+}, \mathrm{N}_{-}\right)=$ $(2916,1458)$ and $(1728,432)$ were considered for asymmetries $\xi=2$ and 4 respectively. The trajectories involved $\approx 10^{6}$ time steps, with time step $\delta t^{*}=\delta t \sqrt{\epsilon /\left(m \sigma^{2}\right)} \approx 0.0005$. Cations and anions were assigned the same mass. Averages for the different properties discussed in this paper were obtained over five independent trajectories.

The simulations of the liquid-vapor interfaces were performed in a prismatic box, with the longest box axis, $z$, perpendicular to the interface plane $x y$. The box length, $L_{z}$ was at least 4 times longer than $L_{x}=L_{y}$. Full periodic boundary conditions were employed in the three directions. The interfacial area, $A=L_{x} L_{y}>(10 \sigma)^{2}$, was large enough to avoid the "periodic error", which can result in large errors in the computation of the surface tension. ${ }^{3}$ The initial configuration for these simulations consisted of a pre-equilibrated liquid slab surrounded by vacuum at a density close to a known coexistence density of the charge asymmetric hard core model. ${ }^{25}$ The final configuration from that simulation was used as initial configuration for subsequent simulations at different temperatures. In addition to the explicit simulation of the liquid-vapor interface we performed constant temperature-pressure (NpT) Molecular Dynamics simulations ${ }^{31,32}$ of the soft core model for charge asymmetries $2: 1$ and 4:1. These simulations were performed to validate the liquid coexistence densities obtained from the explicit interface studies. A typical simulation involved about 500 ions.

Long range Coulombic interactions in both Monte Carlo and Molecular Dynamics simulations were computed using the Ewald summation method. ${ }^{28,29,33}$ For the reciprocal vectors we considered, $k_{x}^{\max }=k_{y}^{\max }=7$ and $k_{z}^{\max }=\left(L_{z} / L_{x}\right) k_{x}^{\max }$ and the convergence parameter, $\alpha$ was set to $6 / L_{x}$. A cutoff of half the box length, $L_{x} / 2$ was used to truncate 
the real part of the Ewald sum. This set of parameters provides an accurate representation of the Coulombic potential, with typical relative errors in the energy of the order of $10^{-6}$. The Ewald method was implemented using "conducting" boundary conditions.

The surface tension of the soft core model was calculated via the mechanical definition of the pressure tensor as an average of two interfaces,

$$
\gamma=\frac{L_{z}}{2}\left[<P_{z z}>-\frac{1}{2}\left(<P_{x x}+P_{y y}>\right)\right]
$$

where $P_{\alpha \beta}$ are the diagonal components of the pressure tensor, see ref. ${ }^{3}$ for details. The reduced surface tension is defined as $\gamma^{*}=\gamma \sigma^{2} / \epsilon$.

Charge and size asymmetry results in a interphase electrostatic potential. ${ }^{4,25}$ The potential drop across the liquid-vapor interface can be computed from the electrostatic field component normal to the interface, $\mathrm{E}_{z}$,

$$
E_{z}(z)=\frac{1}{\epsilon_{0}} \int_{-\infty}^{z} \rho_{q}\left(z^{\prime}\right) d z^{\prime}
$$

where $\rho_{q}\left(z^{\prime}\right)$ is the charge density profile, ${ }^{4}$ defined as the number of charges per unit volume at position $z^{\prime}$. The electrostatic potential, $\phi(z)$, at a particular position, $z$, can then be calculated from, $\phi(z)=-\int_{-\infty}^{z} E\left(z^{\prime}\right) d z^{\prime}$.

The Debye-Hückel theory provides a simple approximation to estimate the interphase potential. The equation for the "Galvani potential" has been derived by Aqua et. al., ${ }^{25}$

$$
\Delta \phi^{*}(T)=\phi_{\text {liq }}^{*}-\phi_{\text {vap }}^{*}=\left(1-\xi^{-1}\right) \ln \left[\frac{\rho_{l}(T)}{\rho_{v}(T)}\right]
$$

where $\Delta \phi^{*}=e \phi / k_{B} T$, is the reduced electrostatic potential, and $\rho_{\alpha}^{*}=\rho_{\alpha} \sigma^{3}$ is the reduced coexistence density of phase $\alpha$. A discussion of the accuracy of the Debye-Hückel approximation to describe the electrostatic potential of charge asymmetric ionic liquids is given below.

\section{Results}

Figure 1 shows the phase diagram for the charge asymmetric systems investigated in this work. The coexistence densities were extracted from an analysis of the simulated density profiles. In agreement with previous studies of charge and size asymmetric ionic 
liquids, ${ }^{3,7,25}$ we find that the asymmetry has a large impact on the critical temperature and density. The critical temperature -normalized by $\xi$-decreases with an increase in charge asymmetry. To quantify this drop in critical temperature we have estimated the critical parameters by fitting the coexistence densities to the law of rectilinear diameters. The critical temperature for asymmetry $\xi=2$ is $T_{c}^{*}=0.046 \pm 0.002$ and the density is $\rho_{c}^{*}=0.115 \pm 0.01$, in good agreement with the results obtained by Panagiotopoulos and Fisher. ${ }^{6}$ For asymmetry $\xi=4$, there is a significant decrease in temperature, $T_{c}^{*}=$ $0.034 \pm 0.002$. This estimate also shows good agreement with the results reported by Camp and Patey ${ }^{20}$ using NpT simulations of the bulk phases.

Aqua et. al. have reported simulation data of the phase diagram of the system $\xi=2$ near the critical region ${ }^{25}$. Their results indicate a significant change in the liquid coexistence densities with respect to RPM case (c.f. Fig. 1). In particular, the liquid coexistence densities were much larger than those of the RPM for temperatures $T^{*}<0.045$. This result does not agree with our simulation data. For the $\xi=2$ system we find that the coexistence densities are only slightly higher than those of the RPM (for $\left.T^{*}<0.035\right)$. Actually both phase diagrams differ very little from each other for most of the temperatures investigated. This observation is in line with other simulations of ionic liquids with both size and charge asymmetry ${ }^{6}$. To test whether the differences between our results and those of ref. ${ }^{25}$ are connected to the use of a soft core model, we performed additional Monte Carlo simulations using the hard core model. These new results show an excellent agreement with our data for the soft core case (c.f. Fig.1), indicating that the small differences in the repulsive contribution of both potentials can be discarded as the origin of the discrepancy. To further validate our simulation results, we have performed NpT simulations of the liquid phase of the soft core model along the zero pressure isobar. This isobar represents a good approximation to the coexistence curve, because the vapor pressure of the ionic liquids investigated here is very low. Again, our NpT data (c.f. Table I) are consistent with the simulations of the explicit interface. Hence, we conclude that our results for the $\xi=2$ are correct. We note that in this work we have performed simulations in continuum space, whereas the results reported in ref. ${ }^{25}$ were obtained using the fine-lattice discretization method. ${ }^{34}$

Figure 2 shows our surface tension results for charge asymmetric ionic liquids. Following the dramatic decrease in critical temperature, charge asymmetry has a large impact on the surface tensions. Interestingly all our surface tensions data show a characteris- 
tic inflection point, or "S" shape. This feature is observed in associating systems, e.g., water and hydrogen fluoride, and it has been discussed as a characteristic of associating liquids. ${ }^{35}$ Otherwise, the high temperature behavior of the surface tensions is consistent with the critical temperatures estimated above (see Fig.2).

Charge asymmetry introduces an asymmetry in the chemical potentials of cations and anions, which results in an interphase potential. We have quantified the potential by computing the charge density along the axis normal to the interface (see eqn.(6)). The potential profiles for two representative temperatures, and for the two asymmetries, $\xi=2$ and 4 are reported in Fig.4. The difference in chemical potential between cations and anions increases with charge asymmetry, this results in a larger charge separation at the interface and an increase in the interphase potential. The interface electrostatic potential essentially follows the density profile, and therefore has the same width as the latter, being of the order of 5-10 $\sigma$ for the systems investigated here. Similar widths were observed in size asymmetric ionic liquids. ${ }^{4}$ The electrostatic field is zero away from the interfacial region, showing that the field is quickly screened in both liquid and vapor phases, despite the strong ionic association found in these systems, particularly in the asymmetry $\xi=4$.

We have analyzed the clustering structure of the liquid and vapor phases by quantifying the coordination number anion-cation as a function of a clustering distance (see Fig.5). We consider that two ions are part of the same cluster if their distance is smaller than certain clustering distance, $R_{c}{ }^{19}$ In the vapor phase we find anion-cation coordination numbers of 2 and 4 , for $\xi=2$ and 4 asymmetries systems respectively, indicative of formation of trimers and pentamers that are globally neutral (see snapshots Fig.6). For the low temperature systems reported in Figure 5 the coordination number in the vapor phase shows to be independent on the clustering distance. This effect is connected to the low vapor pressure, with very little ions in the vapor phase and consequently little interaction between neutral clusters. As expected, this situation changes with increasing temperature, or vapor pressure, which favors cluster dissociation, resulting in a slight dependence of the coordination number with clustering distance (see Fig. 5-top and Fig.6-middle). The coordination number of the liquid phase is low as compared with that of a typical liquid. For clustering distances between $R_{c}=1.1$ and 1.2, for which the vapor is mostly organized in molecular species, the liquid coordination number is only $\approx 5$ and 2 for asymmetries $\xi=4$ and 2 respectively. This indicates that the molecular structuring observed in the vapor phases applies also to the liquid phase. One consequence of the low 
coordination number is the formation of cavities in the liquid phase (see Fig. 6 top for an example). These have been quantified in the case of the RPM (i.e. $\xi=1$ ) model, ${ }^{2}$ our results indicate that this effect may be enhanced by asymmetry. Unlike the vapor phase the coordination number in the liquid phase decreases with temperature (see Fig.5), an observation that is directly linked to the decrease in the liquid coexistence density.

We have used our simulation data to test the accuracy of the Debye-Hückel theory $(\mathrm{DH})$ in predicting the interphase potential (see eqn. (7)). The DH theory describes reasonably well the dependence of the electrostatic potential with temperature for the $\xi=2$ case (see Figure 4). Nonetheless, it becomes very inaccurate for the largest asymmetry, $\xi=4$, where the Galvani potential is underestimated by $50 \%$. The DH theory neglects association effects. These have been considered by Aqua et. al. within a Bjerrum approximation. ${ }^{25}$ This new formulation has the advantage that it captures some physical effects that are expected to be significant in determining the interphase potential, e.g., the dependence of ion association with temperature, which is clearly illustrated in the simulation snapshots (c.f. Fig. 6). As discussed in ref. ${ }^{25}$ the consideration of association leads to a decrease in the interphase potential with respect to the DH approach. On the other hand our results indicate that an "increase" in the interphase potential is necessary to improve the agreement between theory and simulation. Hence the current implementation of the Bjerrum needs to be revised.

\section{Summary}

We have reported molecular dynamics simulations of the liquid-vapor interface of charge asymmetric ionic liquids, with charge asymmetries $\left(z_{+}: z_{-}\right)=(1:-2)$ and (1:-4). By analysis of the liquid and vapor densities away from the coexistence region we have constructed the phase coexistence curves for these systems. In agreement with previous studies we find that the charge asymmetry results in a reduction of the reduced critical temperature. Interestingly, the coexistence curve envelope of moderate asymmetries, (2:-1), changes very little -when represented in reduced units- with respect to the restricted primitive model $(1:-1)$. This result is in contrast with the previous simulations of the coexistence curve of the (2:-1) system, which indicated a larger dependence of the coexistence curve with charge asymmetry. ${ }^{25}$ We have tested the consistency of our results by analyzing the sensitivity of the coexistence curve to the repulsive interaction between 


\section{the ions and to the simulation methodology. These tests confirm the validity of our results.}

Our data for the temperature dependence of the surface tension indicate the existence of an inflection point in this quantity. This seems to be present in all the systems investigated here. This feature has been discussed before as a characteristic of associating systems. The charge asymmetric ionic liquid conform to the notion of associating system. Our cluster analysis shows that most ions are organized in cluster that are globally neutral. The charge distribution at the interface shows nonetheless the existence of charge separation which results from the different chemical potential of cations and anions introduced by the charge asymmetry. The interphase potential, Galvani potential, increases with charge asymmetry. We have shown that the Debye-Hückel theory provides a reasonable theoretical approach to analyze qualitatively the dependence of the electrostatic potential with charge asymmetry. It provides a good estimate of the electrostatic potential of moderate asymmetries (2:-1) including its temperature dependence, but underestimates the electrostatic potential (by about $50 \%$ ) of ionic liquids with larger charge asymmetries $(4:-1)$.

\section{Acknowledgments}

It is a pleasure to dedicate this paper to Professor J.J. Weis on occasion of his retirement. JA and FB would like to thank the Royal Society and La Academia Mexicana de Ciencias for financial support. FB wish to thank the Imperial College High Performance Computing Service for providing computer resources. 


\section{References}

[1] M. González-Melchor, J. Alejandre and F. Bresme, Phys. Rev. Lett., 90, 135506 (2003).

[2] F. Bresme and J. Alejandre, J. Chem. Phys., 118, 4134 (2003).

[3] M. González-Melchor, F. Bresme and J. Alejandre, J. Chem. Phys., 122, 104710 (2005).

[4] F. Bresme, M. González-Melchor and J. Alejandre, J. Phys. Condens.: Matter, 17, S3301 (2005).

[5] J.M. Romero-Enrique, G. Orkoulas, A.Z. Panagiotopoulos and M.E. Fisher, Phys. Rev. Lett., 85, 4558 (2000).

[6] A. Z. Panagiotopoulos and M. E. Fisher, Phys.Rev. Lett., 88, 045701 (2002).

[7] Q.L Yan and J. J. de Pablo, Phys. Rev. Lett. 88, 095504 (2002).

[8] F. W. Tavares, D. Bratko, and J.M. Prausnitz, Curr. Op. Coll. and Interf. Sci., 9, 81 (2004).

[9] Y. Qin and J.M. Prausnitz, J. Chem. Phys., 121, 3181 (2004).

[10] M.E. Leunissen, C.G. Christova, A.P. Hynninen, C.P. Royall, A.I. Campbell, A. Imhoff, M. Dijkstra, R. van Roij and A. van Blaaderen, Nature, 437, 235 (2005).

[11] F. Bresme, C. Vega and J.L.F. Abascal, Phys. Rev. Lett., 85, 3217 (2000).

[12] C. Vega, J.L.F. Abascal, C. McBride, F. Bresme, J. Chem. Phys., 119, 964 (2003).

[13] A.P. Hynninen, M.E. Leunissen, A. van Blaaderen and M. Dijkstra, Phys. Rev. Lett., 96, 018303 (2006).

[14] M. E. Fisher, J. Stat. Phys., 75, 1,(1994); J. Phys.: Condens. Matter, 8, 9103, (1996).

[15] G. Stell, J. Stat. Phys., 78, 197, (1995), J. Phys.: Condens. Matter, 8, 9329, (1996).

[16] H. Weingärtner and W. Schröer, Adv. Chem. Phys., 116, 1, (2001). 
[17] J.L.F. Abascal, F. Bresme and P. Turq, Mol. Phys., 81, 143 (1994).

[18] J.C.G. Montoro, F. Bresme and J.LF. Abascal, J. Chem. Phys., 101, 10892 (1994).

[19] F. Bresme, E. Lomba, J.J. Weis and J.L.F. Abascal, Phys. Rev. E, 51, 289 (1995).

[20] P. J. Camp and G. N. Patey, J. Chem. Phys. 111, 9000 (1999).

[21] M.E. Fisher and Y. Levin, Phys. Rev. Lett., 71, 3826 (1993); Y. Levin and M.E. Fisher, Physica A, 225, 164 (1996).

[22] Q.L. Yan and J. J. de Pablo, J. Chem. Phys, 116, 2967 (2002).

[23] D. W. Cheong and A. Z. Panagiotopoulos, J. Chem. Phys, 119, 8526 (2003).

[24] Y. C. Kim, M. E. Fisher and A. Z. Panagiotopoulos, Phys. Rev. Lett, 95, 065701 (2003).

[25] J.N. Aqua, S. Banerjee and M. E. Fisher, Phys. Rev. E, 72, 041501 (2005).

[26] Y. C. Kim, M. E. Fisher, and A. Z. Panagiotopoulos, Phys. Rev. Lett, 95, 195703 (2005).

[27] W.G. Hoover, Phys. Rev. A, 31, 1695 (1985).

[28] D. Frenkel and B Smit, Understanding Molecular Simulation: From algorithms to applications, Academic Press, (2002).

[29] M.P. Allen and D.J. Tildesley, Computer Simulation of Liquids, Clarendon Press Oxford, (1997).

[30] G. J. Martyna, M. E. Tuckerman, D. J. Tobias and M. L. Klein, Molec. Phys. 87, 1117 (1996).

[31] M. E. Tuckerman, J. Alejandre, R. López-Rendón, A. L. Jochin and G. J. Martyna, J. Phys. A: Math. Gen. 39, 5629 (2006).

[32] S. Melchiona, G. Ciccotti and B. L. Hollian B. L, Mol. Phys, 78, 533 (1993).

[33] P. P. Ewald, Ann. Phys., 64, 253, (1921). 
[34] Y. C. Kim, M. E. Fisher, Comp. Phys. Comm., 169, 295 (2004).

[35] V.C. Weiss, and W. Schroer, J. Chem. Phys., 122, 084705 (2005). 


\section{Figure Captions}

FIG. 1. Liquid-vapor phase diagram of ionic fluids as a function of charge asymmetry. Full line, Molecular dynamics simulations of the SPM model (c.f. eqn. (3)) (1:-1) taken from reference. ${ }^{3}$ The full circle represents the estimated critical point of the SPM model (1:-1). Dashed line, coexistence curve for asymmetry (2:-1) obtained using the fine-lattice discretization method ref. ${ }^{25}$ Squares, Molecular Dynamics simulations of (2:-1) systems using the SPM model. Triangles pointing upwards, Monte Carlo simulations of the (2:-1) asymmetric liquid using a hard core for the repulsive contribution of the potential eqn.(2). Triangles pointing downwards, Molecular Dynamics simulations of the SPM model for charge asymmetry (4:-1).

FIG. 2. Temperature dependence of the surface tension of ionic liquids as a function of charge asymmetry. All the results correspond to the SPM model. The (1:-1) results were taken from Ref. ${ }^{3}$ The critical temperatures are also shown (stars).

Fig. 3. Electrostatic potential of ionic liquids as a function of charge asymmetry and temperature. Left, results for (2:-1) asymmetry, $T / T_{c}=0.76$ full line, and $T / T_{c}=0.87$, dashed line. Right, results for (4:-1) asymmetry, $T / T_{c}=0.74$, full line and $T / T_{c}=0.88$, dashed line.

Fig. 4. The interphase (Galvani) potential of charge asymmetric ionic liquids as a function of temperature. Lines, results from the Debye-Hückel theory (ref. ${ }^{25}$ ), lines and symbols, results from molecular dynamics simulations of the SPM model investigated in this work. Fig. 5 Anion-cation coordination number as a function of clustering distance. Top, asymmetry (1:-2), $\mathrm{T}^{*}=0.035$ (circles) and $\mathrm{T}^{*}=0.043$ (squares). Bottom, asymmetry (1:-4), $\mathrm{T}^{*}=0.0275$. Full symbols represent the coordination number in the liquid phase, and open symbols in the vapor phase.

Fig. 6 Snapshots of the liquid-vapor interface of ionic liquids showing the clustering structure. The lines join ions separated by distances $<1.2 \sigma$. Top, $(1:-2), \mathrm{T}^{*}=0.035$, middle, (1:-2), $\mathrm{T}^{*}=0.043$ and bottom, (1:-4), $\mathrm{T}^{*}=0.0275$. 
TABLE I: Interfacial properties of ionic fluids with different charge asymmetry. In all cases $z_{-}=-1$. The superscript $a$ indicates NpT Molecular Dynamics simulations of bulk liquid at zero pressure.

\begin{tabular}{ccccc}
\hline \hline$z_{+}$ & $T^{*}$ & $\rho_{l}^{*}$ & $\rho_{v}^{*}$ & $\gamma^{*}$ \\
\hline \hline 2 & 0.025 & 0.581 & - & 0.0087 \\
& 0.030 & 0.493 & - & 0.0069 \\
& 0.035 & 0.409 & 0.0002 & 0.0043 \\
& 0.040 & 0.320 & 0.0026 & 0.0021 \\
& 0.043 & 0.262 & 0.0057 & 0.0012 \\
& $0.030^{a}$ & 0.496 & & \\
& $0.036^{a}$ & 0.392 & & \\
& $0.040^{a}$ & 0.325 & & \\
\hline 4 & 0.020 & 0.620 & - & 0.0066 \\
& 0.0225 & 0.579 & 0.0001 & 0.0056 \\
& 0.025 & 0.535 & 0.00036 & 0.0043 \\
& 0.0275 & 0.485 & 0.0017 & 0.0027 \\
& 0.030 & 0.424 & 0.0067 & 0.0015 \\
& $0.02^{a}$ & 0.619 & & \\
& $0.025^{a}$ & 0.538 & & \\
& $0.030^{a}$ & 0.428 & & \\
\hline \hline
\end{tabular}




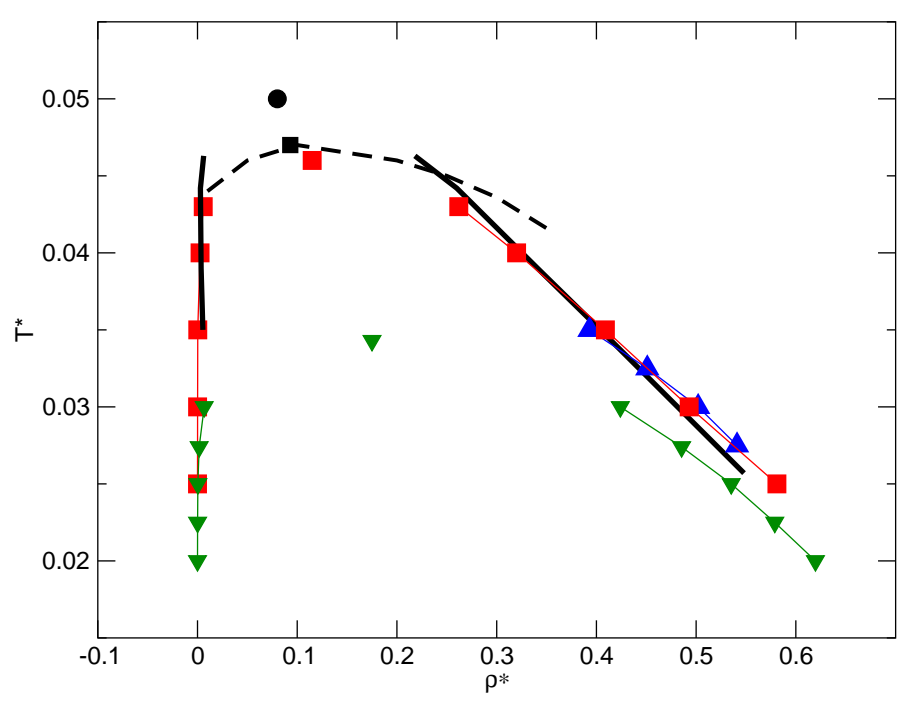

FIG. 1: J. Alejandre, F. Bresme and M. Gonzalez-Melchor, Mol. Phys.

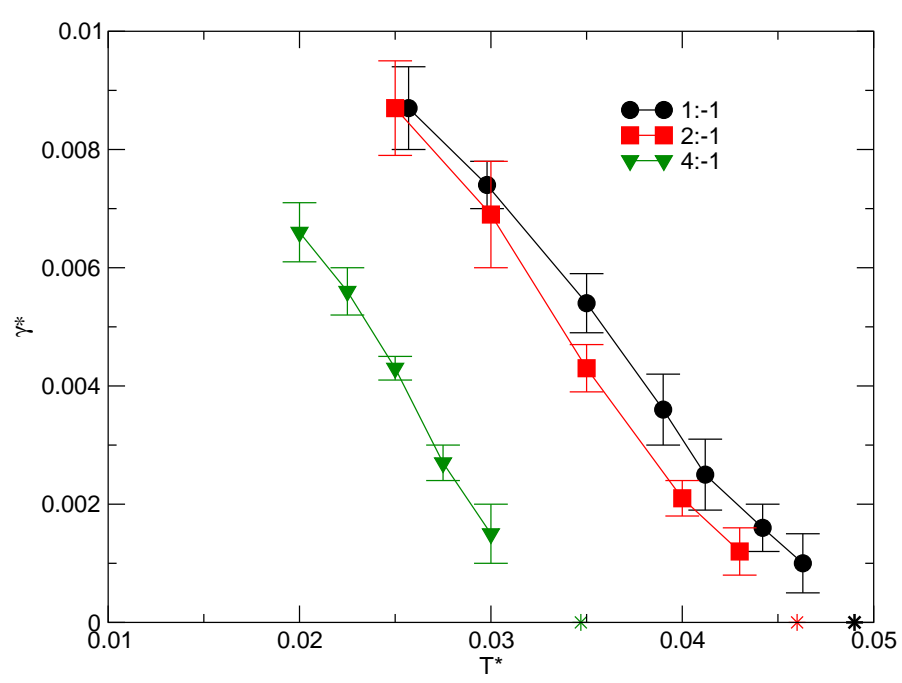

FIG. 2: J. Alejandre, F. Bresme and M. Gonzalez-Melchor, Mol. Phys. 

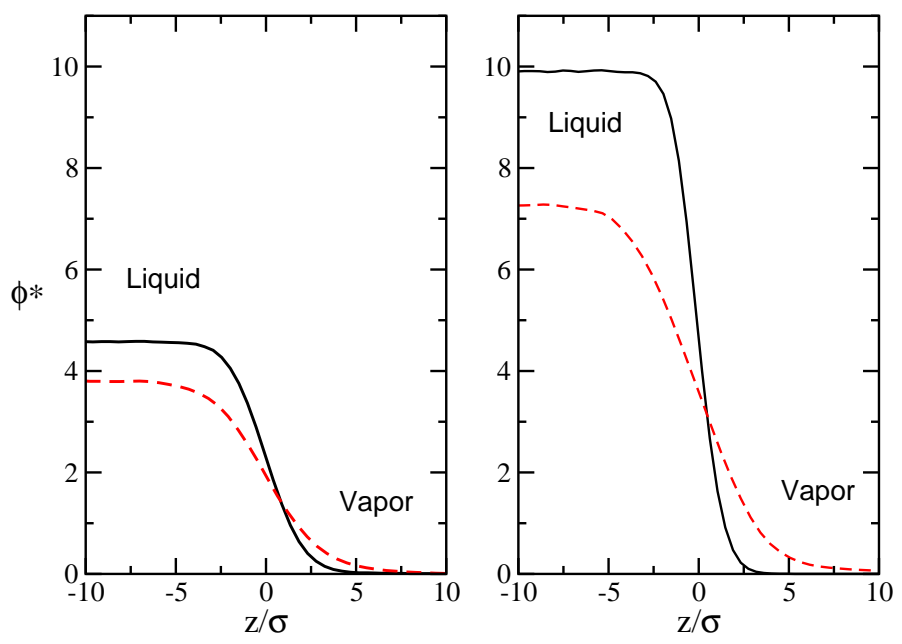

FIG. 3: J. Alejandre, F. Bresme and M. Gonzalez-Melchor, Mol. Phys.

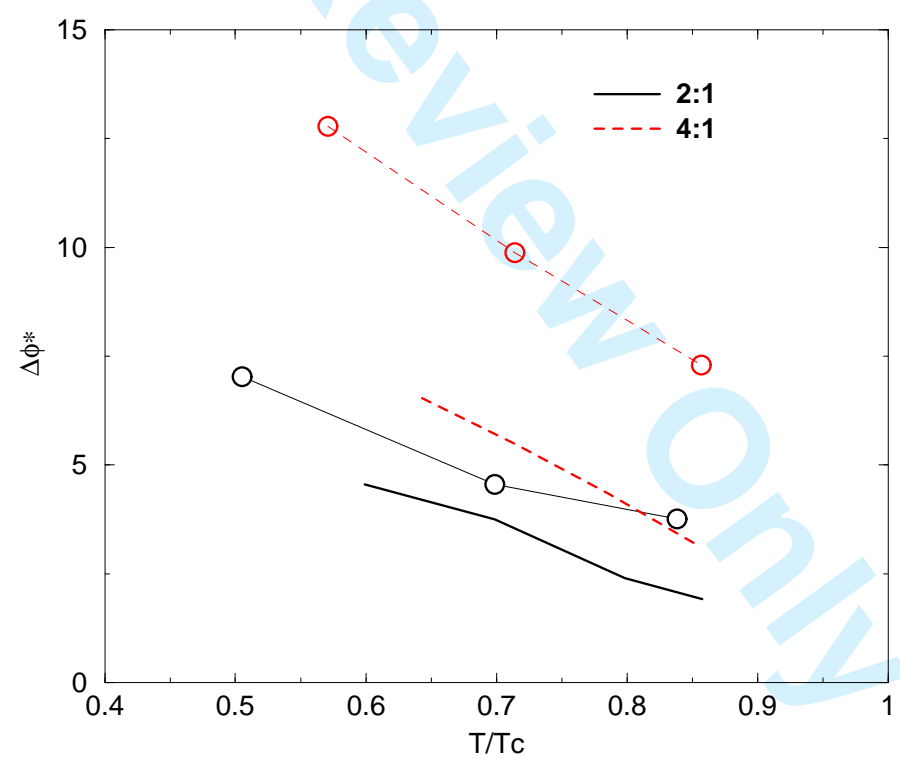

FIG. 4: J. Alejandre, F. Bresme and M. Gonzalez-Melchor, Mol. Phys. 

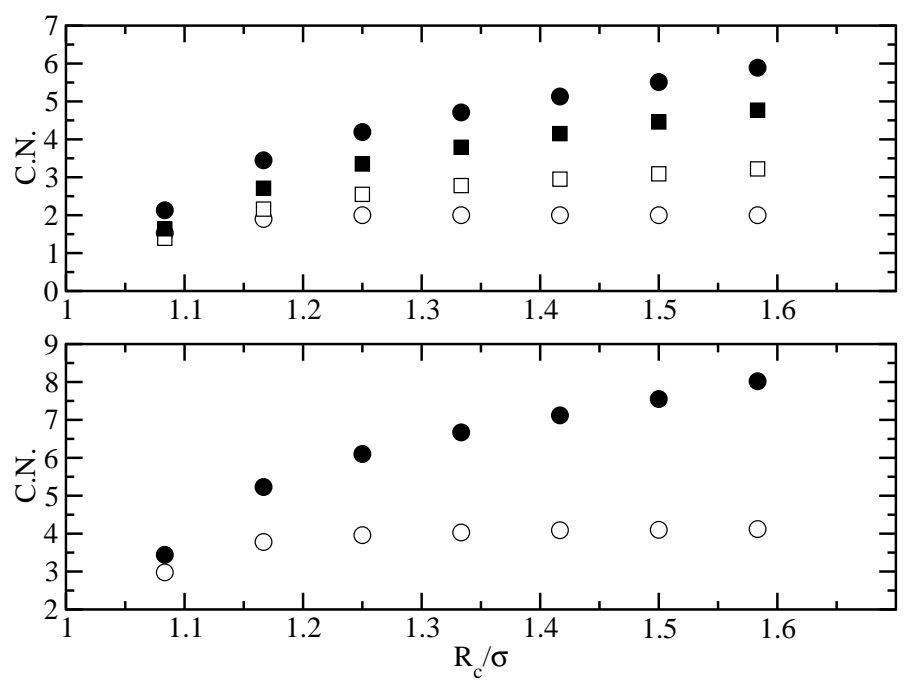

FIG. 5: J. Alejandre, F. Bresme and M. Gonzalez-Melchor, Mol. Phys.
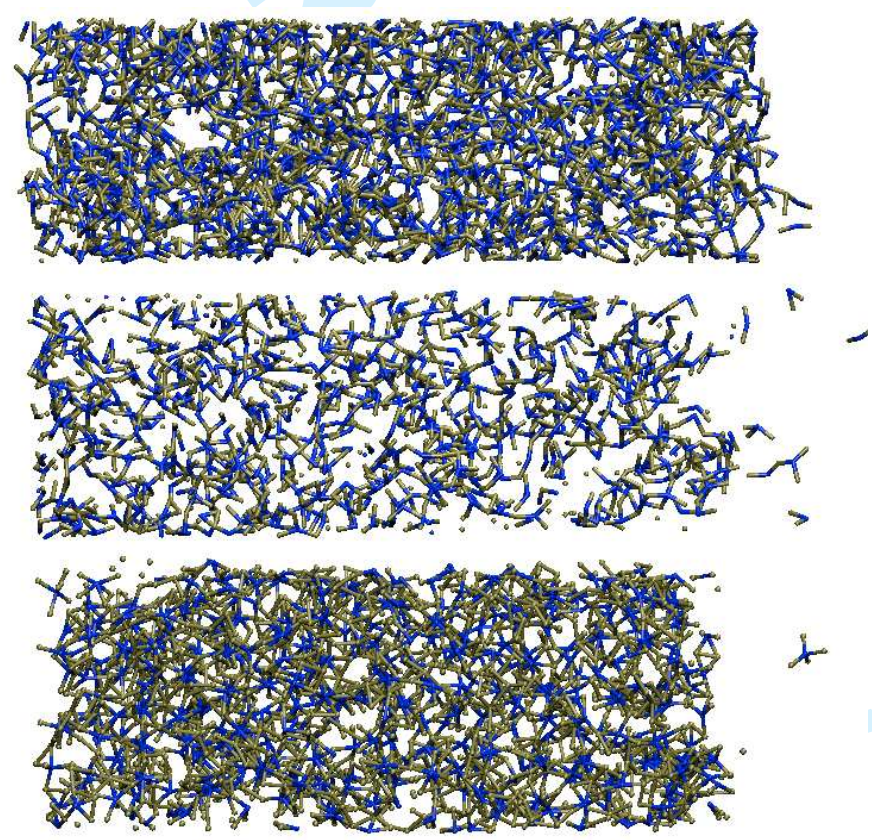

FIG. 6: J. Alejandre, F. Bresme and M. Gonzalez-Melchor, Mol. Phys. 


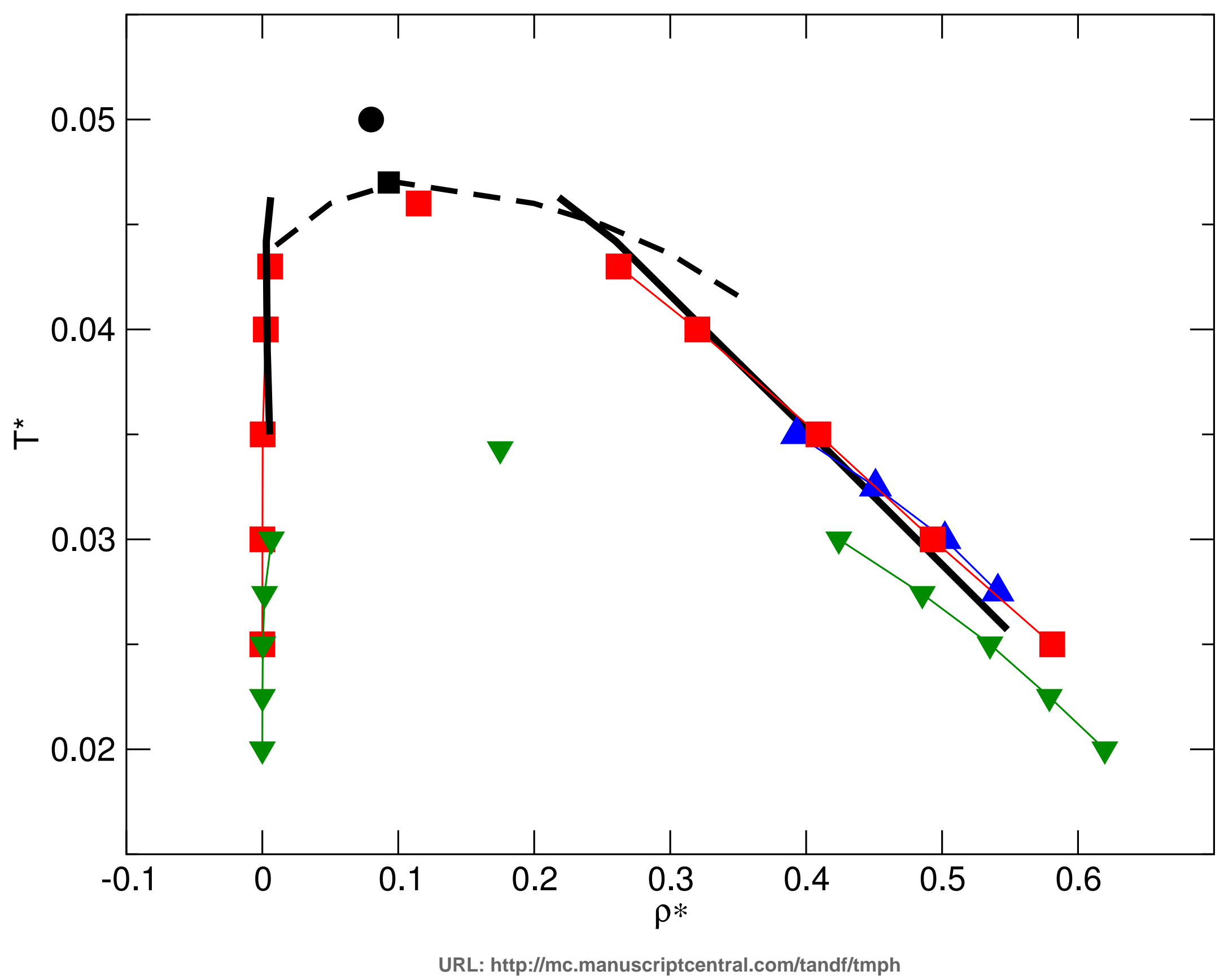




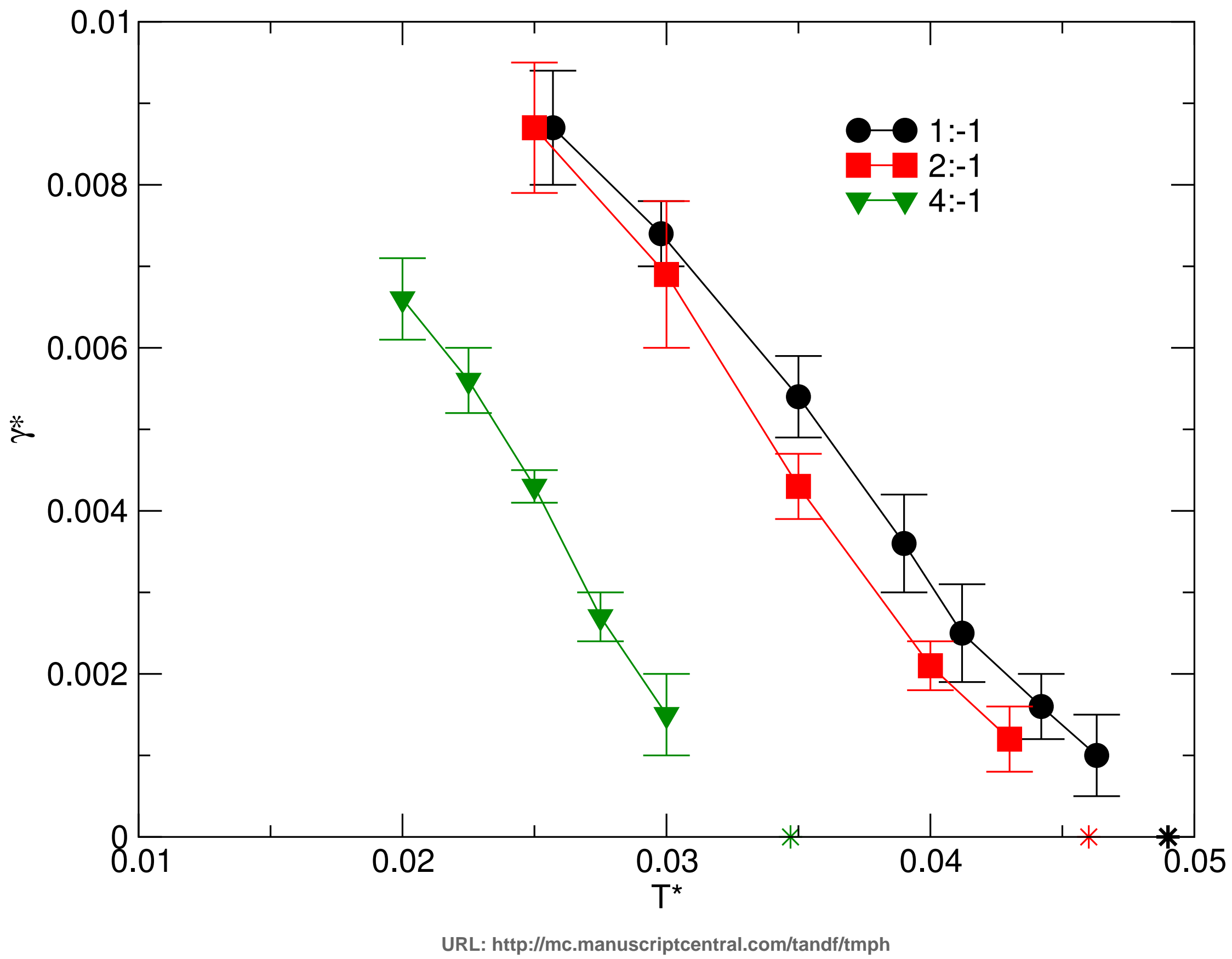




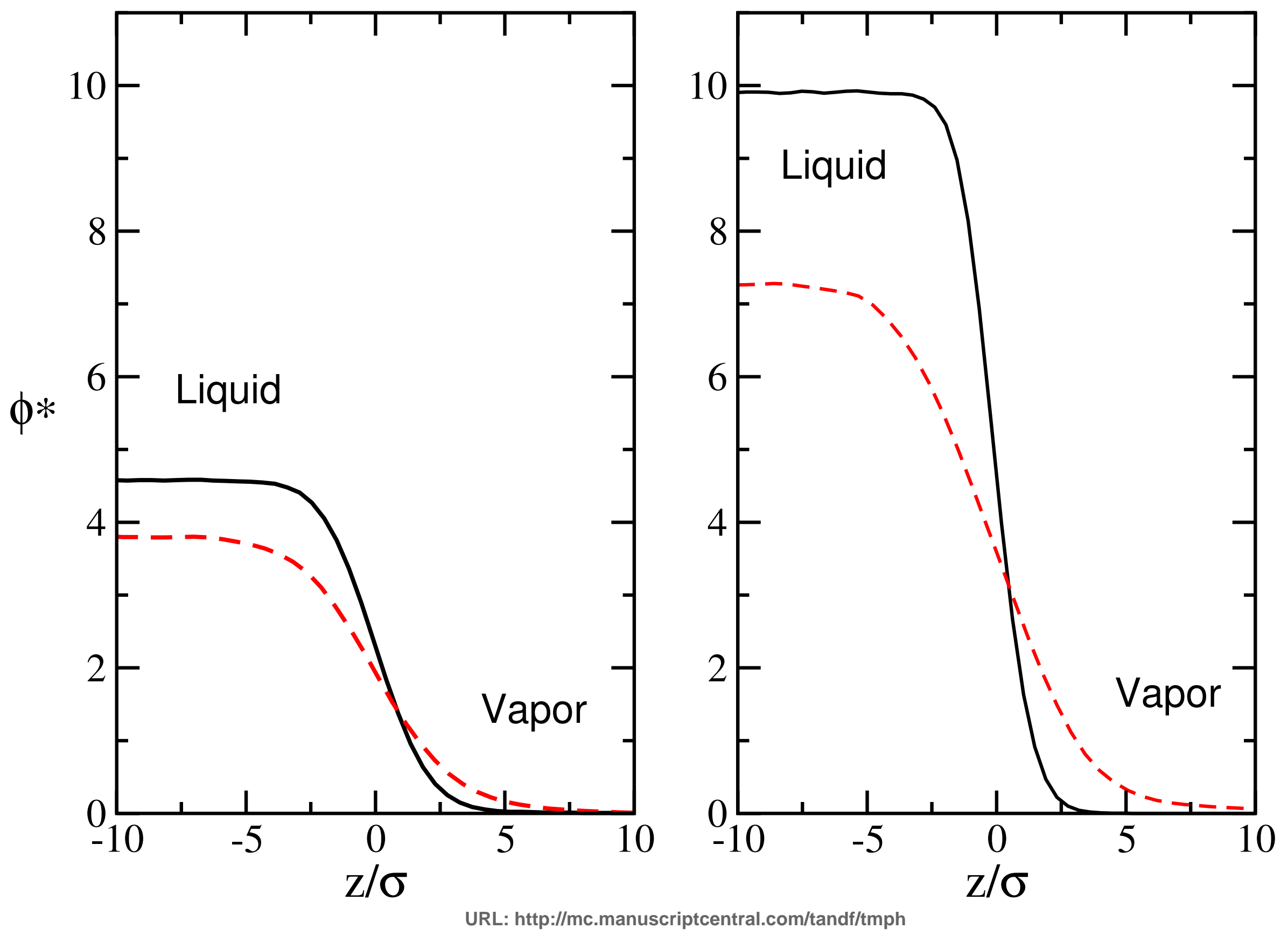




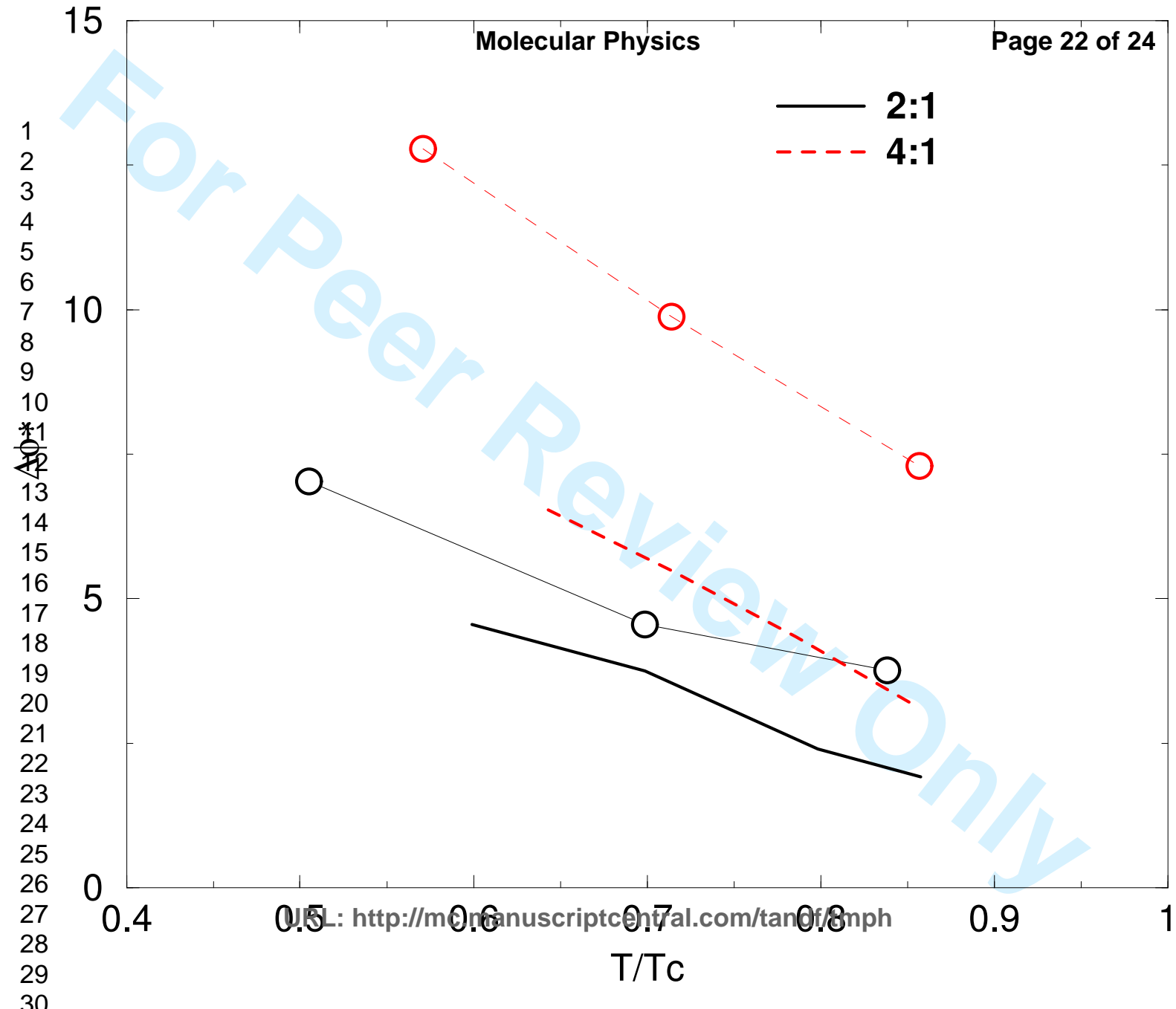



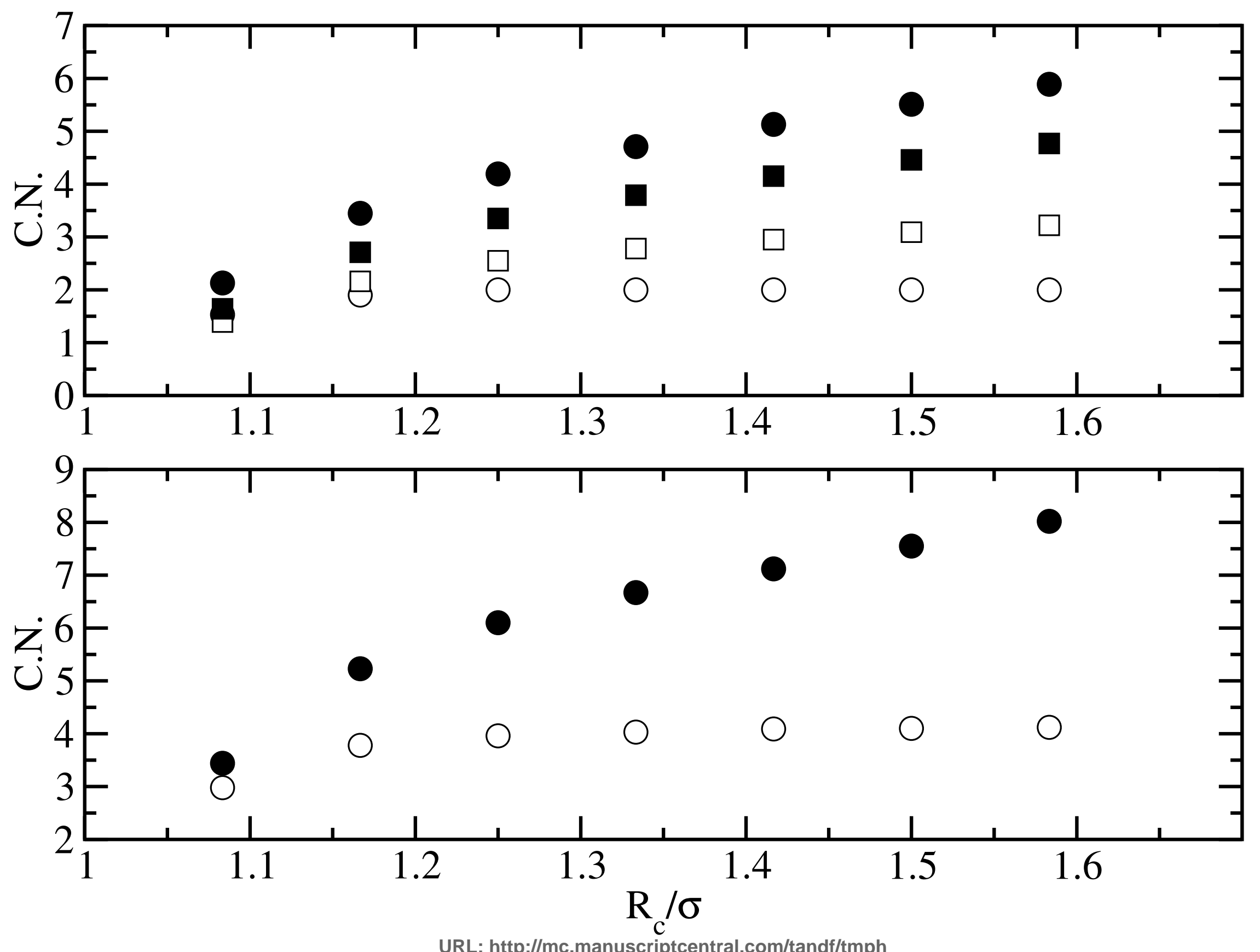

URL: http://mc.manuscriptcentral.com/tandf/tmph 


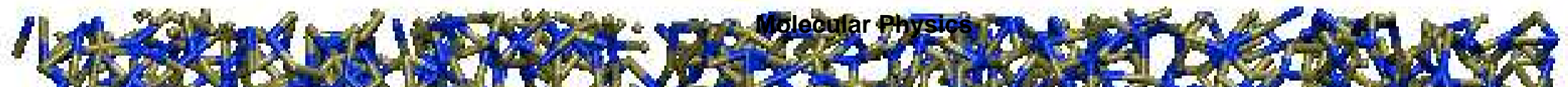

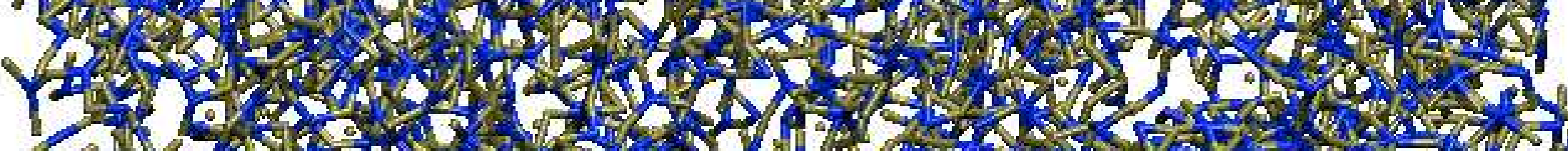

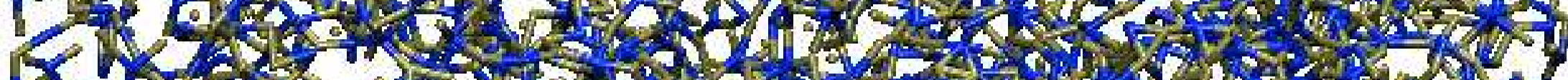

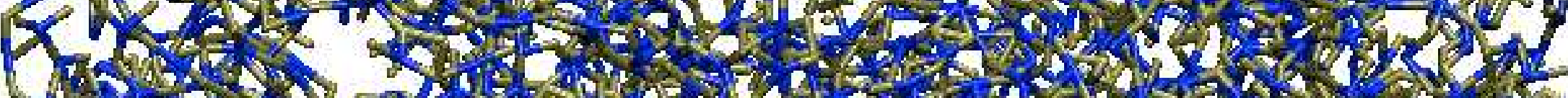

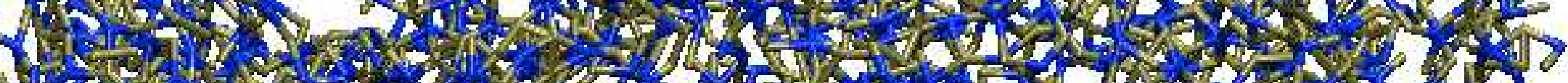
7. H.

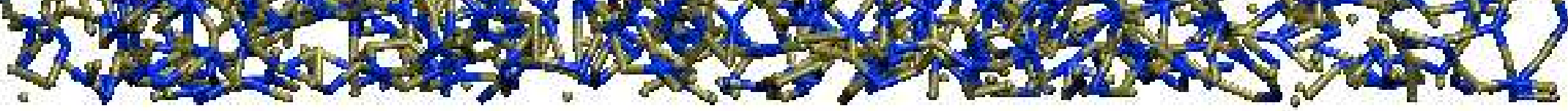

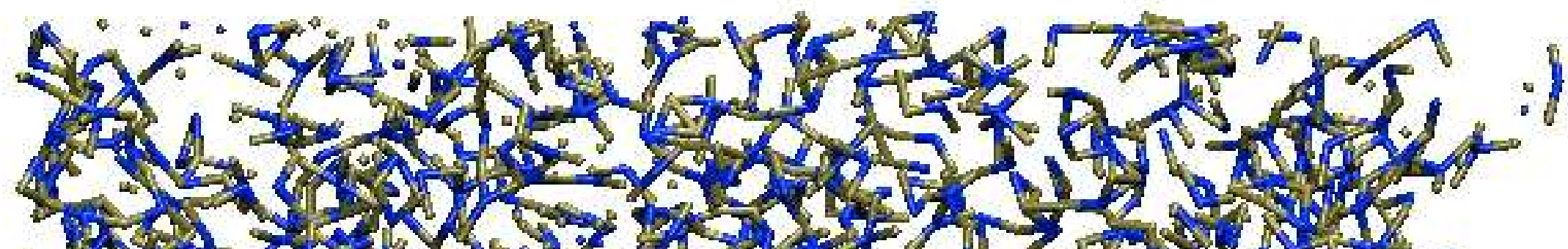

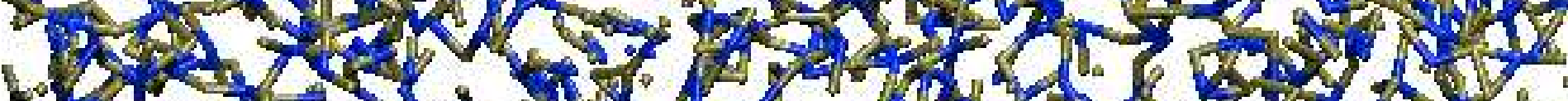

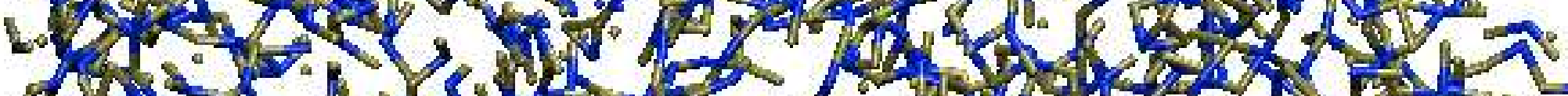

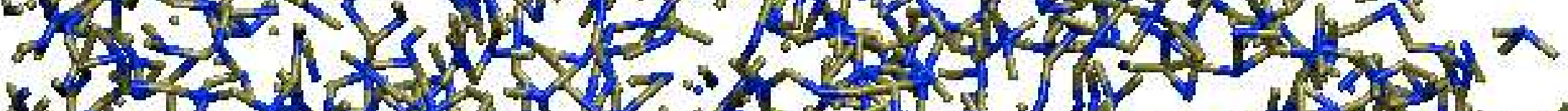

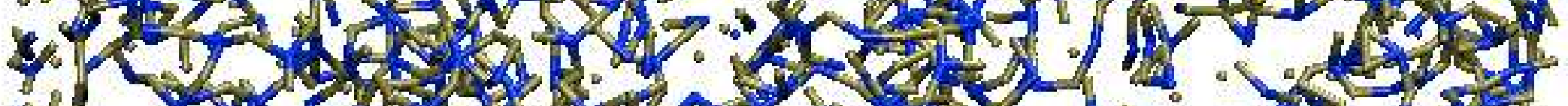
25 . $\Rightarrow$

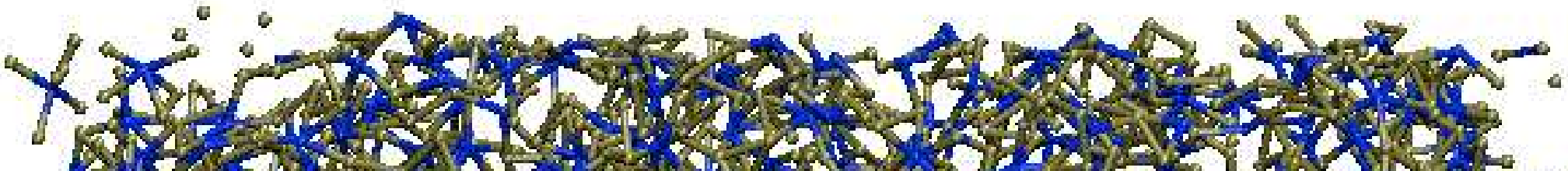

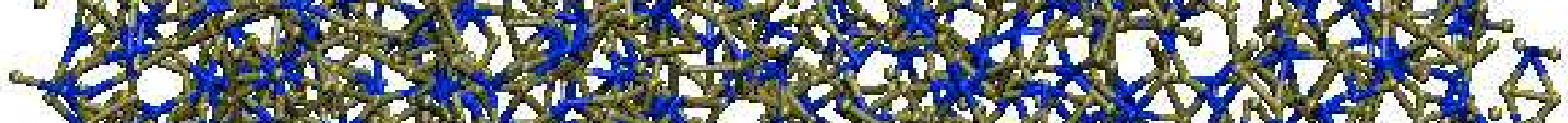

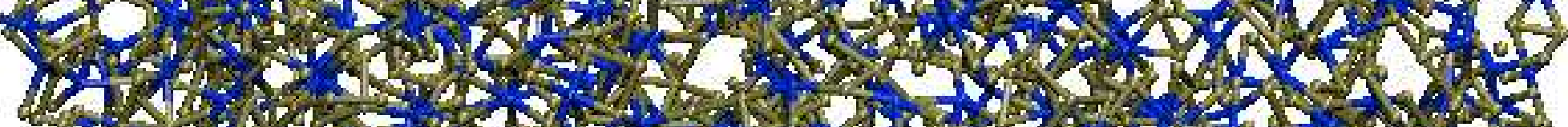

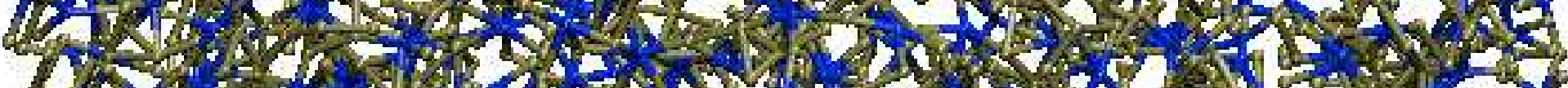

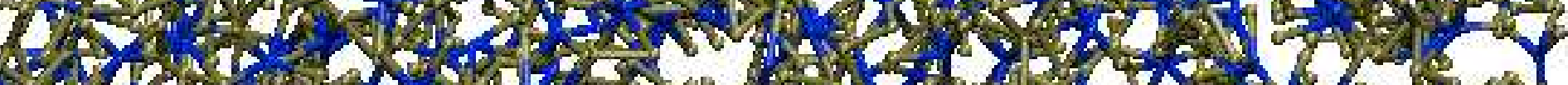

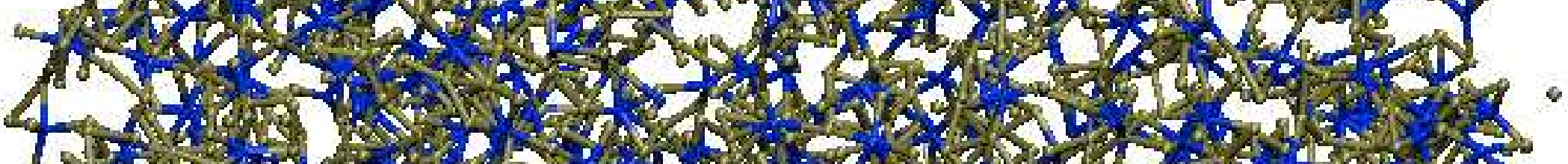

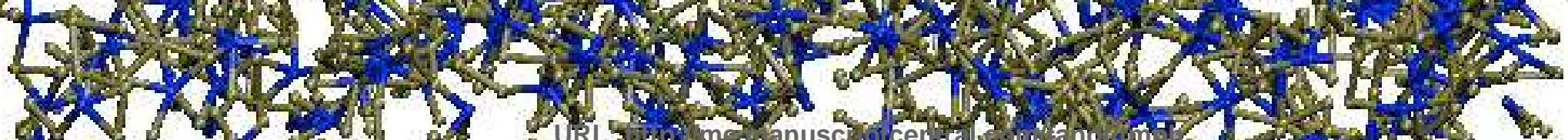

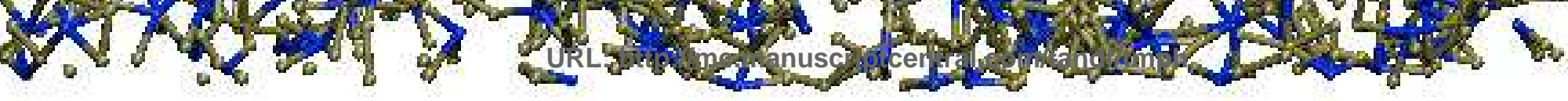

